\title{
Diffusion layers of zinc selenide with a predominant edge emission
}

\author{
${ }^{1}$ Makhniy V.P., ${ }^{1}$ Slyotov M.M., ${ }^{1}$ Tkachenko I.V., ${ }^{1}$ Slyotov A.M., \\ ${ }^{1,2}$ Horley P.P., ${ }^{3}$ Vorobiev Yu.V. and ${ }^{2}$ González-Hernández J. \\ ${ }^{1}$ Yuri Fedkovych Chernivtsi National University, 58012 Chernivtsi, Ukraine, \\ oedpt@chnu.cv.ua \\ ${ }^{2}$ Centro de Investigación en Materiales Avanzados, S.C. (CIMAV) \\ Chihuahua / Monterrey, Miguel de Cervantes 120, 31109 Chihuahua, México, \\ jesus.gonzalez@cimav.edu.mx \\ ${ }^{3}$ Laboratorio de Investigación en Materiales, Centro de Investigación y de \\ Estudios Avanzados del Instituto Politécnico Nacional (CINVESTAV), 76230 \\ Querétaro, México, vorobiev@qro.cinvestav.mx
}

Received: 24.07.2009

\begin{abstract}
Investigations of luminescence in diffusion $\mathrm{ZnSe}$ layers have revealed a pronounced 'blue' band in both $p$ - and $n$-layers doped with the chemical elements of I-VI groups. We have discussed the nature of acceptor centres, luminescent properties and the mechanisms for radiation recombination in the material under study.
\end{abstract}

Keywords: zinc selenide, diffusion layers, point defects, luminescence, recombination.

PACS: 71.20.Nr, 71.55.Gs, 78.40.Fy, 78.55.Et, 78.66.Hf

UDC: 535.37

\section{Introduction}

Physical parameters of zinc selenide make it one of the most promising materials for modern electronics. The band gap of $\mathrm{ZnSe}\left(E_{g} \approx 2.7 \mathrm{eV}\right.$ at $\left.300 \mathrm{~K}\right)$ almost completely covers the visible spectral range, expanding to its blue region. However, a number of technological obstacles complicate development of injection sources for spontaneous and stimulated emission. In particular, construction of the corresponding devices would require materials with high $n$ - and $p$-conductivities that possess high edge luminescence at $300 \mathrm{~K}$ [1]. On the contrary, the now available bulk crystals of $\mathrm{ZnSe}$ are characterised by weak electron conductivity and intense red-orange band in their emission spectrum. The latter band is caused by recombination at the associative centres formed by doubly-charged negative vacancies of zinc $V_{Z n}^{\prime \prime}$ (acceptors) and singly-charged positive vacancies of selenium $V_{S e}^{\bullet}$ (donors) [2]. Any variation of parameters of a material should involve a controlled change of its point defect ensemble. In a wide sense, we can classify these defects as intrinsic (vacancies, interstitial atoms) and impurity defects. Unfortunately, the doping 
of the crystals is insufficient to achieve a required type and magnitude of its conductivity, or to gain light emission in the short-wave spectral range, increase time stability, etc. To solve these problems, one should perform some additional post-growth technological operations.

An alternative approach can also be found aiming to use thin films [3] rather than bulk crystals. The layers of II-VI compounds are usually obtained by annealing of the melt, ion implantation or epitaxy. However, each of these methods has its disadvantages weak solubility of II-VI compounds in low-volatile metals, toxicity of the reagents, high cost or complexity of the equipment, etc. [4]. These disadvantages might be successfully avoided using a diffusion of hetero- and isovalent compositions, a technological approach employed for doping of elementary semiconductors and III-V compounds [5]. The preliminary studies [5] have shown that the method is also applicable to wide-band II-VI compounds. This technological universality makes it a timely and very important task to investigate the technology for diffusion layers of zinc selenide, aiming to obtain the material with high $n$ - and $p$-conductivity and efficient emission in the blue spectral interval, as well as to study their luminescence properties.

\section{Sample preparation and experimental procedure}

The diffusion layers were formed on the basis of non-doped ZnSe crystals grown from the melt in noble gas atmosphere with the Bridgman-Stockbarger technique. The samples had weak electron conductivity $\left(\sim 10^{-10} \Omega^{-1} \mathrm{~cm}^{-1}\right)$ at the room temperature. To perform diffusion, we cut the substrates with dimensions of $4 \times 4 \times 1 \mathrm{~mm}^{3}$ from the crystals obtained and then subjected them to mechanical and chemical polishing in the $\mathrm{CrO}_{3}: \mathrm{HCl}$ solution diluted in the proportion 2:3. The doping was performed from the vapour phase in a closed volume, using several chemical elements from I-VI groups of the periodic table as doping impurities. The substrates of $\mathrm{ZnSe}$ were loaded into quartz containers, pumped out to $10^{-4}$ Torr and sealed. The diffusion process was carried out in isothermal conditions at the temperature $T=1100 \mathrm{~K}$. The doping impurity and the substrates were situated at the opposite ends of the container. The technological cycle was concluded by annealing, resulting in formation of the surface layers with different conductivities. The conductivity was estimated from the measurements of thermal voltage and studies of electro-physical properties of the films.

Ohmic contacts to the samples obtained by us were made by soldering indium to the $n$-type layers and by chemical deposition of copper to the $p$-type material. The depth of electrically-active centres was determined from the plots of resistivity $v s$ temperature. A wide choice of doping impurity elements from the I-VI groups of the periodic system allowed us to obtain the layers of desired thickness and topology, with different conductivity types with respect to the basic crystal.

The spectra of optical reflection, absorption, transmission and luminescence of the resulting material were studied using the universal optical setup, consisting of a diffraction monochromator MDR-23, systems of standard synchronous detection and registra- 
tion, as well as emission sources. Photoluminescence (PL) was excited by a nitrogen laser with the wavelength $\lambda_{\mathrm{m}} \sim 0.337 \mu \mathrm{m}$. The illumination level varied by four orders of magnitude with a set of calibrated filters. The transmission and reflection were studied for a $100 \mathrm{~W}$ halogen lamp producing a continuous spectrum. The measuring complex enabled to study all the required characteristics in both a standard and $\lambda$-modulation modes at the main and doubled frequencies. The application of this technique revealed some singularities in the optical spectra, which would have been unobservable under standard measurements. All the spectra were recorded automatically and corrected according to the error function of the equipment.

\section{Results and discussion}

Doping by the elements of I, II and IV-VI groups from the vapour phase has allowed us to obtain the diffusion layers with intense edge emission. The spectrum of the latter at $T=300 \mathrm{~K}$ lacks any structure and embraces the energies $\hbar \omega \sim 2.4 \div 2.8 \mathrm{eV}$. At the same time, we have found that the luminescence of the diffusion layers features several composite bands formed by different recombination processes. Here we consider the nature and formation mechanisms of defect centres responsible for these transitions. In order to simplify interpretations, the characteristics observed are compared with those of the initial material.

The luminescence and the electro-physical properties of non-doped original $\mathrm{ZnSe}$ crystals are defined by the intrinsic point defects of the material, mainly composed by $V_{S e}^{\bullet}$ and $V_{Z n}^{\prime \prime}$ [2], with the former created by donors compensating hole conductivity. Recombination of electrons localised at Se vacancies with the free holes produces lowintensity emission in the blue region. The main contribution to the luminescence spectrum is thus made by transitions of the associative centres $\left(V_{S e}^{\bullet} V_{Z n}^{\prime}\right)$ determining the intense red-orange band with the maximum at $\hbar \omega_{\mathrm{m}} \sim 1.98 \mathrm{eV}[2,5]$ (see Fig. 1).

Doping of ZnSe crystals with the elements of I and V groups leads to formation of diffusion layers with a characteristic predominant intense emission in the blue region of the spectrum and inversion of material conductivity. The luminescence spectra of these layers at $77 \mathrm{~K}$ contain several components denoted here by symbols $A, B$ and $C$ (see Fig. 1) for the case of doping elements of $\mathrm{V}$ group, and with the letters $D, E$ and $F$ for the doping elements of I group. The dominating bands $B$ and $E$ result from doping with the aforementioned impurity elements. These bands are defined by emissive transitions at the acceptor centres, which are caused by replacements of $\mathrm{ZnSe}$ in the cation and anion sublattices. In the case of doping with the elements of $\mathrm{V}$ group, simple acceptor replacement centres $A s_{S e}, S b_{S e}$ and $B i_{S e}$ are formed [6], which is proved by electron paramagnetic resonance (EPR). According to our electro-physical studies, the ionisation energy of these centres comprises $110 \div 130 \mathrm{meV}$, which corresponds to the position of $\hbar \omega_{\mathrm{m}}$ maxima of the band $E_{g}-\hbar \omega_{m}+k T / 2$ with a precision of $k T$. At the same time, even this "elementary 
band" is characterised by asymmetric spectrum with an abrupt high-energy end and smoothly-sloping low-energy side, as shown in Fig. 2 for the particular case of $\mathrm{ZnSe:Bi}$ layers.

These luminescence spectra are characterised with $\hbar \omega_{\mathrm{m}}$ independent of the excitation level $L$, while the increase of the latter is accompanied with growing half-width $\hbar \omega_{1 / 2}$ of the spectra and the emission intensity in the low-energy spectral region. These properties are characteristic for the carrier recombination through the local centres under phonon interaction [7]. According to the Yuls-Kreger model, the total luminescence spectrum obeys the law

$$
N_{\omega}=\sum_{n=0}^{\infty} N_{\omega_{n}} \exp \left[-\frac{\left(\hbar \omega-\hbar \omega_{m} \pm n \hbar \omega_{0}\right)^{2}}{\sigma^{2}}\right]
$$

where the sign " +" corresponds to absorption of phonon with the energy $\hbar \omega_{0}$, and "_" to its emission.

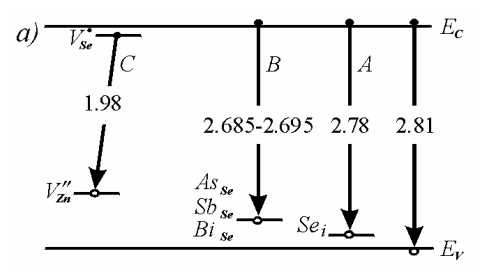

a

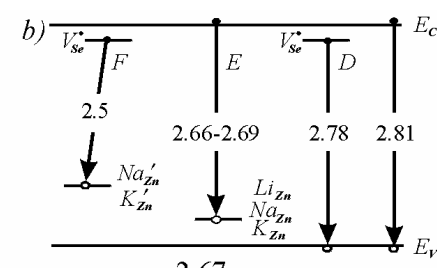

$\mathrm{b}$

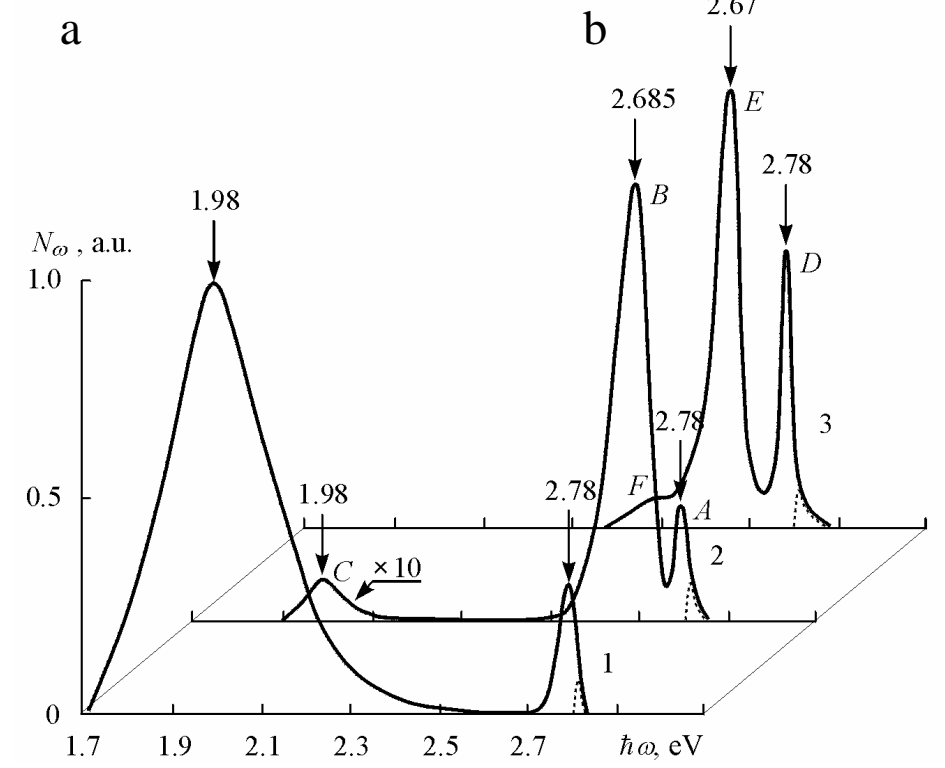

Fig. 1. Intensity-normalised PL spectra for the initial ZnSe crystals (curve 1) and diffusion layers of ZnSe:Bi (curve 2) and ZnSe:K (curve 3) with $p$-type of conductivity. The illumination intensity is $10^{15}$ photons per second and the temperature $T=77 \mathrm{~K}$. The upper panel shows band diagrams for the emissive transitions in $\mathrm{ZnSe}: \mathrm{Bi}(\mathrm{a})$ and $\mathrm{ZnSe}: \mathrm{K}$ (b) layers. 


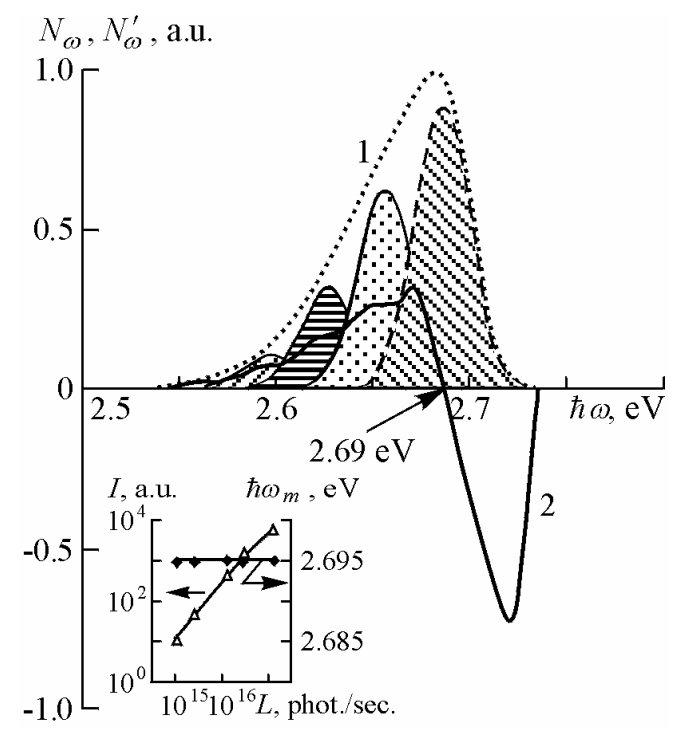

Fig. 2. Standard (curve 1) and differential (curve 2) PL spectra for the diffusion ZnSe:Bi layers. The components are calculated according to Eq. (1). The inset shows positions of the maxima and intensity of the impurity band as functions of excitation level for $T=77 \mathrm{~K}$.

The parameter $\sigma$ is connected with the band half-width as

$$
\sigma=\hbar \omega_{1 / 2} /(8 \cdot \ln 2) \text {, }
$$

with the amplitude of the $n$-phonon band given by

$$
N_{\omega_{n}}=N_{\omega_{0}} \bar{M} / n !
$$

Here $\bar{M}$ is a degree of electron-phonon interaction determined by the ratio $N_{\omega_{1}} / N_{\omega_{0}}$. As seen from Fig. 2, the calculated bands generally provide an acceptable explanation of the experimental spectrum. The positions of the phonon bands in the differential spectra measured with $\lambda$-modulation method correspond to equidistant peaks $\left(\hbar \omega_{0}=31 \mathrm{meV}\right)$. The main band is caused by electron recombination taking place between the conduction band and deep acceptor levels formed by $A s_{S e}, S b_{S e}$ and $B i_{S e}$ centres. These mechanisms determine the $p$-type conductivity of the diffusion layers obtained by us.

The hole conductivity may be also caused by interstitial atoms of $\mathrm{Se}_{\mathrm{i}}$ formed upon diffusion of $\mathrm{V}$ group elements [6]. This process leads to appearance of the $A$ band in the luminescence spectra, for which the difference of the energies,

$$
E_{g}-\hbar \omega_{m}+k T / 2=(2.70-2.68+0.013) \mathrm{eV}=0.033 \mathrm{eV},
$$

is close to the activation energy of a shallow acceptor level of $\mathrm{Se}_{\mathrm{i}}$. The emissive transitions via this level would form a dominating illumination edge. The intensity suppression observed for the orange band $\left(\hbar \omega_{m} \approx 1.98 \mathrm{eV}\right)$ is caused by decreasing $V_{S e}^{\bullet}$ concentration, which occurs as a consequence of "healing" of $V_{S e}$ by the elements of V group. This phe- 
nomenon decreases the concentration of donor-acceptor pairs $\left(V_{Z n}^{\prime \prime} V_{S e}^{\bullet}\right)$, which are responsible for recombination causing the orange emission. It is worthwhile that $V_{S e}^{\bullet}$ vacancies compensate the hole conductivity of $\mathrm{ZnSe}[2,6]$, decreasing the concentration of holes and imposing a conductivity reversal.

The luminescence of $\mathrm{ZnSe}$ diffusion layers obtained under the doping with the elements of I group is defined by a dominating band $E$ (see Fig. 1). This band is formed by the transitions via acceptor centres $L i_{Z n}, N a_{Z n}$ and $K_{Z n}$, which are generated upon replacement of cations in zinc selenide sublattice. The differential optical transmission spectra $T_{\omega}^{\prime}$ and the temperature dependence of the conductivity have enabled determining the position of the acceptor levels as $120-150 \mathrm{meV}$, which increases for the case of impurities with higher atomic weights. The diffusing atoms "heal" the vacancies of zinc. Being introduced in smaller concentration, they eventually cause extinction of the red-orange emission (Fig. 1), since they represent a second partner in the associative centres $\left(V_{Z n}^{\prime \prime} V_{S e}^{\bullet}\right)$ responsible for the band mentioned above.

Diffusion of the impurity atoms into the crystalline lattice introduces deformations. In the first approximation, this effect appears due to difference of covalent radii of the doping atoms $(1.32-1.98 \AA)$ and the zinc ones $(1.27 \AA)$. The deformation process is accompanied by generation of vacancies in the selenium sublattice. The donors are formed in the singly-charged state $\left(V_{S e}^{\bullet}\right)$ and recombination of the localised electrons with the free holes induces the edge emission of the diffusion layers (Fig. 1). The generation of $V_{S e}$ has been indirectly confirmed by annealing in the mixed vapours of $\mathrm{Li}$ and $\mathrm{Se}$ [8], which leads to "healing" of the vacancies and intensity quenching for optical bands due to lowering concentration of the corresponding recombination centres. During this process one also observes increase in the $p$-type conductivity of $\mathrm{ZnSe}: \mathrm{Li}, \mathrm{Se}$ layers, in comparison with $\mathrm{ZnSe}: \mathrm{Li}$, as a consequence of lower concentration of the compensating donors $V_{S e}^{\bullet}$.

The edge emission of the diffusion layers doped with the atoms of I group (the same concerns to the elements belonging to $\mathrm{V}$ group) contains two main bands attributed to recombination involving shallow donor $\left(V_{S e}^{\bullet}\right.$, with $\left.E_{d} \approx 30 \mathrm{meV}\right)$ and deep acceptor levels (with $E_{a} \sim 0.12-0.15 \mathrm{eV}$ ). $\lambda$-modulation studies of their luminescence spectra have allowed clear separation of the components mentioned (see Fig. 3). The differential curves $N_{\omega}^{\prime}$ at low energies intersect the abscissa at different points, which determine the positions of acceptor centres in $\mathrm{ZnSe}$. The equidistant bands correspond to electron-phonon interaction processes at the deep $L i_{Z n}, N a_{Z n}$ and $K_{Z n}$ centres. The distance among these bands points to the energy of longitudinal optical phonon in ZnSe. In the high-energy region, all the $N_{\omega}^{\prime}$ curves intersect the energy axis at a single point, proving the existence of single recombination centres of the type of $V_{S e}^{\bullet}$, which are responsible for the observed emission according to the Klasens scheme for the $p$-type layers. 


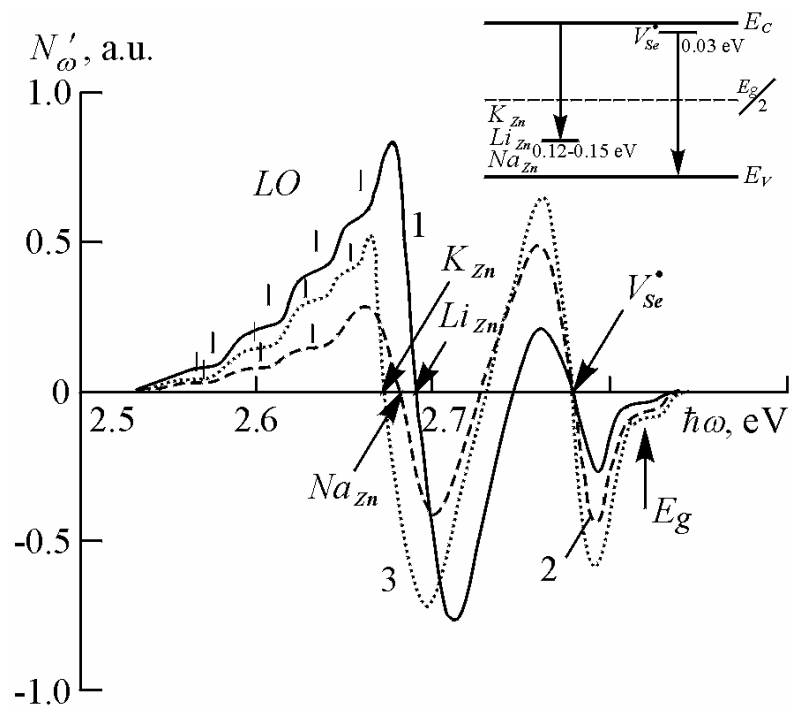

Fig. 3. $\lambda$-modulated PL spectra for the layers ZnSe:Li (curve 1), ZnSe:Na (curve 2) and ZnSe:K (curve 3). Light flux is $L=10^{16}$ photons per second and the temperature $T=77 \mathrm{~K}$. The inset shows band diagram of the light emitting transitions.

The presence of oppositely charged centres in the diffusion $\mathrm{ZnSe}: \mathrm{K}$ and $\mathrm{ZnSe}: \mathrm{Na}$ layers activates formation of donor-acceptor pairs $\left(N a_{Z n}^{\prime} V_{S e}^{\bullet}\right.$ or $\left.K_{Z n}^{\prime} V_{S e}^{\bullet}\right)$. The optical transitions of carriers between the partners of such the associates determine the nature of the emission band $F$ (see Fig. 1). This band has low intensity due to a low concentration of singly-charged donor-acceptor pairs, which is about $3 \div 4$ orders of magnitude lower than that of doubly-charged defects [9]. In general, the edge emission of the diffusion layers based on $\mathrm{ZnSe}$ crystals and formed by doping with the elements of I group can be completely explained by the transitions of carriers shown in Fig. 1 (see the inset b).

The diffusion layers of $\mathrm{ZnSe}$ doped with the elements of I and $\mathrm{V}$ groups can emit light quanta with $\hbar \omega \geq E_{g}$, which is proved by a singularity observed in the corresponding differential spectra $N_{\omega}^{\prime}$ (see Fig. 3). The position of this singularity does not depend on the excitation level $L$ and features the temperature dependence of $\hbar \omega_{\mathrm{m}}$ similar to that of $E_{g}(T)$. Such the properties are inherent for the inter-band recombination of free carriers [10]. In this case the spectral distribution of the emitted emission is given by

$$
N_{\omega}=(\hbar \omega)^{2} \sqrt{\hbar \omega-E_{g}} \exp \left[-\frac{\hbar \omega-E_{g}}{k T}\right] .
$$

The spectral curves calculated with Eq. (4) agree well with the experimental data (see Fig. 1). It is worth special mentioning that the inter-band luminescence is observed 
for every ZnSe sample obtained with the diffusion method. Therefore all the following spectra, including the edge emission area, feature this component, too.

In addition to the case of diffusion with the elements of different valences, the efficient blue edge emission can also be achieved with isovalent impurities and, in particular, magnesium (see Fig. 4). This luminescence would be evoked by exciton nature of a predominant emission [11], which results in a red shift of $\hbar \omega_{\mathrm{m}}$ maxima upon increasing $L$ and the luminescence intensity dependence $I \sim L^{1.5}$ (see the inset in Fig. 4). These features are also characteristic of annihilation of excitons upon inelastic scattering at free carriers [12]. The emission of the diffusion layers of $\mathrm{ZnSe}: \mathrm{Mg}$ includes a whole set of properties, such as anomalously high temperature stability up to $500 \mathrm{~K}$ [13]. This peculiarity is explained by a significant localisation degree of carriers at the isovalent impurities, when compare with that for the impurity atoms with different valence. A modern physical model used in this case is based on the assumption about short-range potential of isovalent impurities [14]. Then the charge redistribution is accompanied by local deformation of crystalline lattice, which triggers generating intrinsic point defects. The nature of the latter determines the conductivity type of the material. In particular, the diffuse layers of $\mathrm{ZnSe}$ doped with isovalent $\mathrm{Mg}$ impurity become a $p$-type material. For determining the type of the corresponding intrinsic lattice defects we have performed calculations based on the concept of effective charge $Q^{*}[14,15]$, permitting to involve interstitial selenium defects $\mathrm{Se}_{\mathrm{i}}[16]$. These acceptor centres should be responsible for the $p$-type conductivity of $\mathrm{ZnSe}: \mathrm{Mg}$ layers, for which the dominating edge emission of excitons is typical.

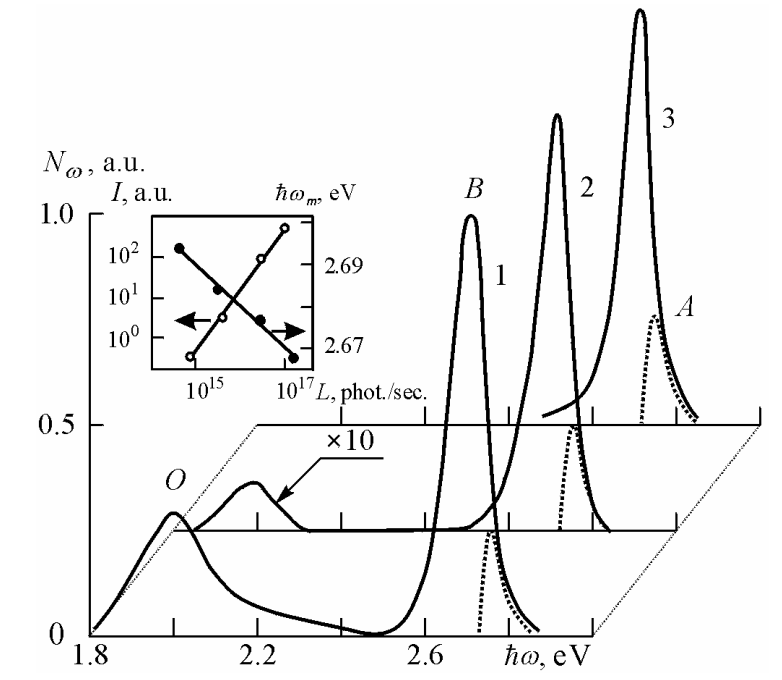

Fig. 4. Intensity-normalised PL spectra measured at $T=300 \mathrm{~K}$ for the initial crystals $\mathrm{ZnSe}(1)$ and the layers obtained by doping with $\mathrm{Zn:Mg}$ in the proportion 1:0 (curve 2) and 0.5:0.5 (curve 3 ). $A$ is a compound band caused by inter-band recombination.

Beyond any doubt, the most important feature that concerns possible applications of diffusion layers is suppression of their red-orange emission characterised with 
$\hbar \omega_{\mathrm{m}} \approx 1.98 \mathrm{eV}$, owing to such isovalent doping impurity as Mg (see Fig. 4). Our studies of the long-wave absorption edge and $\lambda$-modulated reflection have revealed no changes in either $E_{g}$ or the character of optical transitions, which suggests direct-band nature of the material, proving at the same time that no solid solutions $\mathrm{Mg}_{\mathrm{X}} \mathrm{Zn}_{1-\mathrm{x}} \mathrm{Se}$ are formed, at least for the case of large $\mathrm{x}$ values $[13,16]$.

Using the similar diffusion methods, it is possible to obtain $\mathrm{ZnSe}$ layers with higher electron conductivity, when compare to that of the initial crystals. This improvement may be reached by doping the material with the chemical elements of IV group. In particular, using the amphoteric impurity of $\mathrm{Sn}$, we have produced the $n$-type conductivity of $1 \div 10 \Omega^{-1} \mathrm{~cm}^{-1}$ [17]. It is determined by the donors $S n_{Z n}$ formed during the replacement processes in the cation sublattice accompanied with generation of $V_{S e}$. The temperature dependence of the conductivity $\sigma_{n}(T)$ facilitates determination of positions of these levels $(\sim 10 \mathrm{meV}$ and $30 \mathrm{meV}$, respectively). The amphoteric character of the doping impurity also results in replacement process taking place in the anion sublattice and producing the acceptor centres $S n_{S e}$ with the ionisation energy of $\sim 70 \mathrm{meV}$. However, the concentration of the latter is much lower than that of the donor levels, thus explaining a prevalent $n$-type conductivity of the material. At the same time, all the impurity centres contribute significantly to the edge emission observed in the $\mathrm{ZnSe}$ layers.

Contrary to the non-doped zinc selenide, the emission of the diffusion layers with amphoteric impurities is in general characterised with high intensity and can be observed in the short-wavelength region only (for the photon energy $\hbar \omega \geq 2.4 \mathrm{meV}$ ). Our measurements performed at low temperatures ( $77 \mathrm{~K}$ or somewhat higher) have enabled resolving at least three bands, tentatively denominated as $B_{1}, B_{2}$ and $B_{3}$ (see Fig. 5). The band $B_{1}$ has the spectral dependence which is well approximated by Eq. (4) derived for the interband recombination of free carriers. The extrapolation of low-energy end of $B_{1}$ towards the photon energy axis yields a value of $2.81 \mathrm{eV}$. This agrees well with the band gap of $\mathrm{ZnSe}$ at $77 \mathrm{~K}$. The position of the band $\hbar \omega_{\mathrm{m}}$ does not depend on the excitation level $L$, while the band intensity is quadratic in it $\left(I \sim L^{2}\right)$.

The component $B_{2}$ manifests rather different properties. Similar to $\mathrm{ZnSe}: \mathrm{Mg}$ layers, the dependence of the luminescence intensity can be described with the power law $I \sim L^{1.5}$, with $\hbar \omega_{\mathrm{m}}$ being red-shifted for increasing $L$. These properties are characteristic of emission of excitons upon their inelastic scattering at free carriers [12]. It is to be noticed that the difference between $E_{g}$ and $\hbar \omega_{\mathrm{m}}$ for the $B_{2}$ band is less than $14-20 \mathrm{meV}$, thus agreeing well with the binding energy for the free exciton $\left(E_{F E} \approx 16-24 \mathrm{meV}\right.$ for $\mathrm{ZnSe}$ [2]). This is why we are inclined to suggest that the band $B_{2}$ under discussion is associated with the free excitons.

The peak position for the third band $B_{3}\left(\hbar \omega_{\mathrm{m}} \approx 2.74 \mathrm{eV}\right.$ at $\left.T=77 \mathrm{~K}\right)$ is independent of $L$, which is peculiar for the recombination via local centres. Taking into account the 


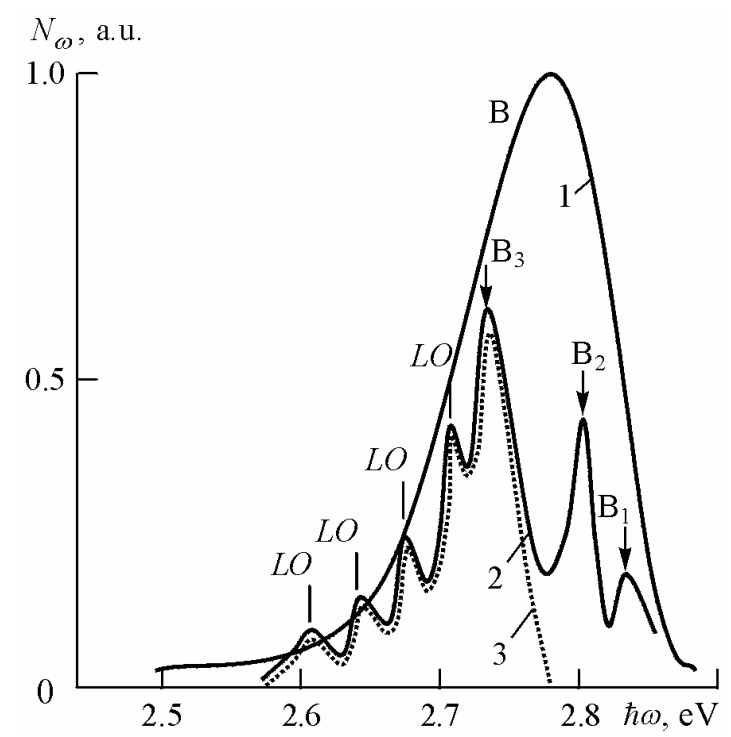

Fig. 5. PL spectra of the diffusion layers $\mathrm{ZnSe}: \mathrm{Sn}$ measured at $T=77 \mathrm{~K}$ under excitation levels $L=10^{18}$ (curve 1) and $10^{16} \mathrm{~kW} / \mathrm{s}$ (curve 2). Curve 3 shows the spectrum calculated according to Eq. (1).

difference between $E_{g}$ and $\hbar \omega_{\mathrm{m}}(\sim 70 \mathrm{meV})$, one can assume that the light emitting transitions involve the centres formed by $S n_{S e}$. Moreover, under certain illuminations $L$ it is possible to resolve several equidistant bands in the low-energy region, which are separated roughly by $\sim 30 \mathrm{meV}$ intervals. This agrees with the energy of $L O$-phonon. Generally, the total spectrum of this component can be explained in framework of the YulsKreger model and Eq. (1), similarly to the situation with the diffusion layers obtained by doping with the elements of I and V groups. This proves strong electron-phonon interaction of the local centres. Its degree $\bar{M}$ determined from Eq. (3) enables us to calculate the shape of the band $B_{3}$. Using the experimental parameters $\bar{M}=0.81$ and $\sigma^{2} \approx 1.2 \cdot 10^{-3} \mathrm{eV}$, we have calculated the band contour with Eq. (2). The calculations have given a result that fits well the experimental curve, if one assumes the emission of four $L O$-phonons (see curve 3 in Fig. 5).

Our results for the luminescence of the diffusion $\mathrm{ZnSe}$ layers highlight significant prospects for the applications of this material. In the first place, the layers obtained by us are very promising owing to their blue emission which, according to our estimations, has the efficiency of $\eta \approx 4-8 \%$. The latter value may be considerably improved up to $\eta \geq 20 \%$, when isovalent doping impurities (in particular, $\mathrm{Mg}$ ) are used. Such an efficiency boost might be ascribed to peculiar properties of isoelectron states in semiconductors $[14,18]$. As already mentioned above, the layers are characterised by a high carrier localisation at the isovalent impurities, which should in turn lead to both temperature and emission stabilities. The intensity of the exciton luminescence increases practically linearly with increasing temperature, while its absolute value decreases only twice on reach- 
ing $500 \mathrm{~K}$ [12]. Let us remark that the isovalent impurities also cause increasing emission stability of the luminescence parameters and the characteristics of material [18].

One of the most important conclusions concerns a possibility for controlling the conductivity type and its magnitude in case of the diffusion layers obtained while doping them with the elements of I-VI groups. This fact opens a wide road to creation of efficient $p$ - $n$-junction capable to emit blue light and characterised with high temperature stability.

\section{Conclusions}

Doping of zinc selenide with the chemical elements of different groups of the periodic table using the vapour phase has allowed us to obtain layers with comparatively high $n$ and $p$-conductivities. The optimal choice of the elements has enabled variation of conductivity type of the resulting materials, so that one can get the layers of desired thickness, which are capable of emitting in the blue spectral region. This emission is formed by several bands contributing to total spectrum differently, depending on the doping impurity used. In general, the edge emission originates from recombination of charge carriers at the local centres and inter-band transitions, as well as annihilation of the excitons. The diffusion layers obtained by us have good prospects for practical applications. They allow generation of photons with the energy $\hbar \omega \geq 2.4 \mathrm{eV}$ and are capable to work in the stimulated emission regime.

\section{Acknowledgments}

This work was partially supported by the budget funding of the Ministry of Education and Science of Ukraine and the Projects No 15-82 (State Registration Number 0109U002240) and No SU/447-2009 (State Registration Number 0109U005913).

\section{References}

1. Vavilov V S, 1994. Peculiarities of the physics of wide-band semiconductors and their applications. Uspekhi Fiz. Nauk. 164: 287-295.

2. Nedeoglo D D and Simashkevich A V, Electric and luminescent properties of zinc selenide. Chisinau: Stiintsa (1984).

3. Alferov Zh I, 1998. The history and the future of semiconductor structures. Fiz. Tekhn. Poluprov. 32: 3-19.

4. Pichugin I G and Tairov $\mathrm{Yu}$ M, Technology of semiconductor devices. Moscow: Vysshaya Shkola (1984).

5. Makhniy V P, Arhilyuk L I, Grivul V I, Mel'nyk V V, Slyotov M M, Sobistchanskiy B M and Tkachenko I V, 2006. Prospects of using the wide bandgap II-VI compounds of in short-wave sensors. Sensor Electron. Microsyst. Techolog. 3: 30-34.

6. Makhiniy V P, Slyotov M M and Chaban Yu Ya, 2000. Hole conductivity in the crystals of zinc selenide doped with the elements of $\mathrm{V}$ group from the vapour phase. Pis'ma v Zhurn. Tekhn. Fiz. 26: 13-16.

7. Medvedev S A, Ed. Physics and chemistry of AIIBVI. Moscow: Mir (1970). 
8. Makhniy V P, Slyotov M M and Chaban Yu Ya, 1998. p-ZnSe and p-ZnS crystals emitting in dark-blue and UV spectral regions. Functional Materials. 5: 31-35.

9. Arhilyuk L I, Makhniy V P, Slyotov M M, Gorley V V and Tkachenko I V, 2007. Mechanisms of defect formation for $\mathrm{ZnSe}$ with isovalent oxygen impurity. Telecommunication and Radio Engineering. 66: 465-471.

10. Gribkovskiy V P, Theory of light absorption and emission in semiconductors. Minsk: Nauka i Tekhnika (1975).

11. Slyotov M M, 2001. Edge luminescence from zinc selenide doped with isovalent magnesium impurity. Techn. Phys. Lett. 27: 63-64.

12. Koh Era and Langer D W, 1970. Luminescence of ZnSe near the band edge under strong laser light excitation. J. Lumin. 1-2: 514-527.

13. Makhniy V P, Slyotov A M and Chaban Yu Ya, 2002. Effect of magnesium isovalent impurity on the temperature stability of blue luminescence in zinc selenide. Techn. Phys. 47: 786-787.

14. Fistul' V I, Atoms of the doping impurity in semiconductors (state and behaviour). Moscow: Fizmatlit (2004).

15. Ryzhikov V D, Scintillation crystals of the semiconducting AIIBVI compounds. Obtaining, properties, and applications. Moscow: NIITEKhIM (1989).

16. Makhniy V P and Slyotov A M, 2004. Optical properties of ZnSe:Mg diffusion layers. Ukr. J. Phys. Opt. 5: 136-140.

17. Grivul V I, Makhniy V P and Slyotov M M, 2007. The nature of the edge luminescence of the diffusion layers ZnSe:Sn. Fiz. i Tekhn. Poluprov. 41: 806-807.

18. Makhniy V P, Slyotov M M, Demych N V and Slyotov A M, Peculiarities of the physical properties of the heterolayers of isovalent replacement of the wide-band II-VI compounds. Abstract booklet of the International scientific conference (Minsk, Belarus, 2005), pp. 385-387.

Makhniy V.P., Slyotov M.M., Tkachenko I.V., Slyotov A.M., Horley P.P., Vorobiev Yu.V. and González-Hernández J., 2009. Diffusion layers of zinc selenide with a predominant edge emission Ukr.J.Phys.Opt. 10: 206-217.

Анотація. Досліджено люмінесценцію шарів ZnSe, одержаних за методом дифузії. Показано, що існує иілий набір елементів I-VI груп, які дають змогу одержати шари $n$ - та $p$ типу провідності з ефективним випромінюванням в синьо-голубій області спектру. Розглянуто природу акцепторних иентрів, пояснено люмінесцентні властивості, а також механізми випромінювальної рекомбінації. 